\title{
Super-Earths give theorists a super headache
}

\section{An abundance of medium-sized worlds is challenging planet-formation models.}

\section{BY ERIC HAND}

B y now, it's not surprising that NASA's Kepler space telescope is turning up extrasolar planets by the bushel. Last week, at the first Kepler science conference at NASA's Ames Research Center in Moffett Field, California, mission scientists announced that the space telescope has identified 2,326 candidate planets, nearly doubling its haul since February.

But what has puzzled observers and theorists so far is the high proportion of planets - roughly one-third to one-half - that are bigger than Earth but smaller than Neptune. These 'super-Earths' are emerging as a new category of planet - and they could be the most numerous of all (see 'Super-Earths rising'). Their very existence upsets conventional models of planetary formation and, furthermore, most of them are in tight orbits around their host star, precisely where the modellers say they shouldn't be.

"It poses a challenge," says Douglas Lin, a planet-formation modeller and director of the Kavli Institute for Astronomy and Astrophysics at Peking University in Beijing, China. "You can't just tweak the parameters. You need to think about the physics."

Guided by the example of our own Solar System, with its distinct sets of large and small worlds, early planet-formation models were based on the notion of 'core accretion'. Dust swirling around a star in a protoplanetary disk can aggregate into small planetesimals of rock and ice, which collide and stick together. The inner part of the disk contains too little material for these cores to grow much bigger than Earth. But farther out, they can attain ten Earth masses or more, enough to attract a vast volume of gas and become Jupiter-like.

The detection, starting in 1995, of Jupitersized planets with orbits as short as a few Earth days contradicted these models. The theorists revised their models to allow these hot Jupiters' to form far from their star and then migrate in. Yet these models predicted that anything reaching super-Earth size should either become a gas giant or be swallowed by its star, creating a 'planetary desert' in this size range. Kepler's discoveries wreck those predictions. "It's a tropical rainforest, not a desert," says Andrew Howard, an astronomer at the

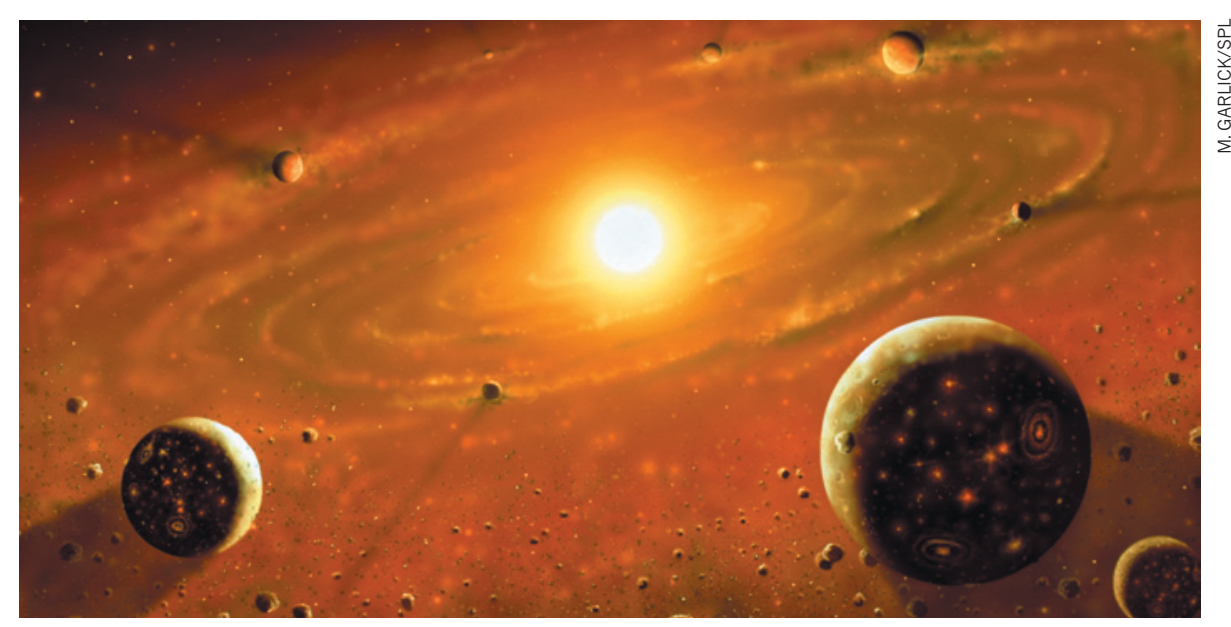

Planets coalesce out of dust swirling around their parent stars - but where super-Earths form is unclear.

University of California, Berkeley. "We hope the theory is going to catch up."

Kepler measures a planet's size by detecting how much light it blocks as it passes in front of its star. For a handful of the super-Earths detected by Kepler, ground-based observations have also determined mass, by tracking the wobble of the host star induced by the planet's gravity. And some of these super-Earths seem to have very low densities - indicating that they may have small rocky cores surrounded by large gas envelopes.

Kepler astronomer Jack Lissauer, of Ames, thinks that they may have begun as small cores

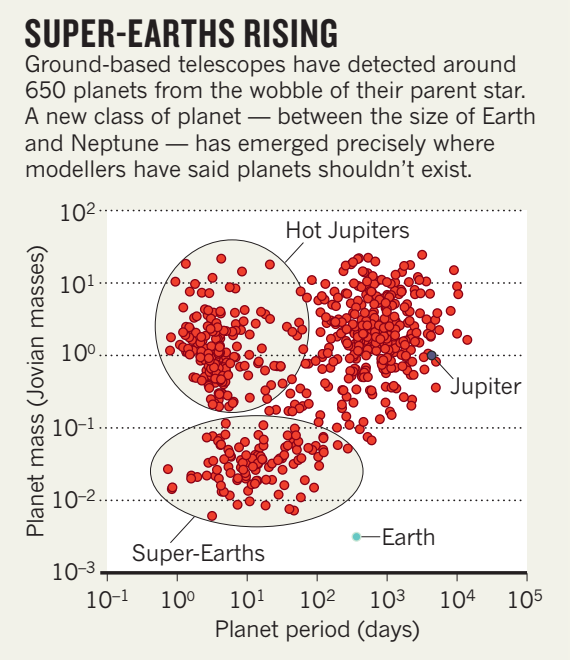

in the outer parts of their solar system, accreting a large amount of gas without reaching the point of runaway growth that leads to a true gas giant. Without the gravitational heft of a giant to hold in gas, such a planet would have a large, low-density atmosphere, but it could still grow to super-Earth size by a cooling process that shrinks the atmosphere and allows more gas to be drawn in, he says.

But that scenario may not explain smaller and denser super-Earths. Several such planets have already been detected, and Kepler is starting to reach the sensitivity required to spot them, says Greg Laughlin, an astronomer at the University of California, Santa Cruz. "Kepler is just seeing the tip of the iceberg."

Nor can any current theory explain how super-Earths can sit so close to their stars. Lissauer says the problem lies in the migration portion of the models. But Norm Murray, an astrophysicist at the University of Toronto, is exploring other ways of forming super-Earths. Instead of assembling them and migrating them towards the star, Murray's model first migrates rocky planetesimals and then allows them to accrete. "Migration then assembly' is the catchphrase," he says.

In any event, Laughlin says that modellers will probably find a way to explain the current observations. "They'll scramble to fix the models," he says. But it's probably not the last time they'll have to revisit their codes, he adds. "My prediction is that they'll completely miss the next big thing, whatever that will be." - 